\title{
EHMTI-0195. Cortical changes in medication-overuse headache
}

\author{
F Riederer ${ }^{1 *}{ }^{*}$, M Schaer ${ }^{2+}$, AR Gantenbein ${ }^{3}$, R Luechinger ${ }^{4}$, L Michels $^{5}$, S Kollias ${ }^{5}$, PS Sandor ${ }^{3}$ \\ From 4th European Headache and Migraine Trust International Congress: EHMTIC 2014 \\ Copenhagen, Denmark. 18-21 September 2014
}

\section{Introduction}

In medication-overuse headache $(\mathrm{MOH})$ pain modulation is probably dysfunctional at cortical and subcortical level, resulting in disequilibrium between pain inhibition and facilitation. Volumetric grey matter changes have been found in cortical regions, but also in the brainstem [2], the latter being reversible after successful detoxification [1].

\section{Aims}

Surface-based morphometric analyses should complement volumetric findings, providing more specificity in the metric affected (thickness vs. gyrification). Whereas cortical thickness alterations probably rely on altered trajectories of cortical maturation or neurodegenerative processes, cortical folding (gyrification) abnormalities are thought to reflect early alterations to brain development.

\section{Methods}

In the present study we investigated cortical thickness and gyrification in 29 patients with $\mathrm{MOH}$ according to International Headache Society criteria and 29 age- and gender matched controls, using FreeSurfer. Correction for multiple comparisons was performed.

\section{Results}

In patients with $\mathrm{MOH}$ cortical thickness was decreased in the left middle frontal gyrus (rostral part) compared to controls, whereas local gyrification was increased in the right fusiform gyrus and adjacent temporal regions, as well as in the right occipital pole.

\section{Conclusions}

Decreased cortical thickness in frontal regions corresponds to decreased grey matter volume in similar regions. Increased local gyrification in the right fusiform gyrus corresponds to increased grey matter volume in the previous volumetric study. Increased gyrification in occipital regions might be related to increased susceptibility for cortical spreading depression.

\section{Authors' details}

'2nd Neurological Department, Neurological Center Rosenhuegel, Vienna, Austria. ${ }^{2}$ Stanford University Medical Center, Stanford Cognitive \& Systems Neuroscience Laboratory, Stanford Palo Alto CA 94304, USA. ${ }^{3}$ Neurology, Rehaclinic Bad Zurzach, Bad Zurzach, Switzerland. ${ }^{4}$ Institute for Biomedical Engineering, Swiss Federal Institute of Technology, Zurich, Switzerland. ${ }^{5}$ Institute of Neuroradiology, University Hospital Zurich, Zurich, Switzerland.

Published: 18 September 2014

References

1. Riederer F, et al: J Neurosci 2013, 33(39):15343-15349.

2. Riederer F, et al: The World Journal of Biological Psychiatry 2012, 13(7):517-525.

doi:10.1186/1129-2377-15-S1-E30

Cite this article as: Riederer et al.: EHMTI-0195. Cortical changes in medication-overuse headache. The Journal of Headache and Pain 2014 15(Suppl 1):E30.

\footnotetext{
† Contributed equally

${ }^{1}$ 2nd Neurological Department, Neurological Center Rosenhuegel, Vienna,

Austria

Full list of author information is available at the end of the article
}

\section{SpringerOpen $^{\odot}$}

(c) 2014 Riederer et al; licensee Springer. This is an Open Access article distributed under the terms of the Creative Commons Attribution License (http://creativecommons.org/licenses/by/2.0), which permits unrestricted use, distribution, and reproduction in any medium, provided the original work is properly cited. 